\title{
Analyse de la conversion de l'énergie solaire en matière sèche par un peuplement de luzerne (Medicago sativa L.) soumis à un déficit hydrique
}

\author{
J.L. Durand ${ }^{1}$, G. Lemaire ${ }^{1}$, G. Gosse ${ }^{2}$ et M. Chartier ${ }^{2}$ \\ ${ }_{1}$ INRA, Ecophysiologie des plantes fourragères, 186600 Lusignan; \\ 2 INRA, Bioclimatologie, 78850 Thiverval-Grignon, France
}

(reçu le 30 septembre 1988, accepté le 23 mars 1989)

\begin{abstract}
Résumé - Cinq repousses de luzerne exploitée en fauche ont été étudiées avec ou sans irrigation. Un modèle simple de productivité en fonction de l'énergie interceptée par le couvert végétal est utilisé pour analyser les différents effets de la sécheresse. La prise en compte de la biomasse des souches et de la fraction des pivots située dans les 10 premiers centimètres montre que la répartition de la matière sèche entre parties aériennes et parties souterraines explique au moins $80 \%$ des effets de la sécheresse sur le niveau de production de la culture. Lorsque le déficit s'installe au début de la repousse, la limitation du développement de la surface foliaire entraîne en plus un déficit d'énergie interceptée. C'est dans ces circonstances que la sécheresse se montre la plus pénalisante.
\end{abstract}

consommation en eau - irrigation - indice foliaire - rendement - fourrage - biomasse végétale

Summary - Analysis of solar energy conversion into dry matter by a lucerne crop (Medicago sativa L.) exposed to water deficit. Five regrowth cycles of a lucerne crop irrigated or not were studied over two years. $A$ simple growth model is used to analyse the drought's effects.

The main feature of the model consists of the analysis of the intercepted solar radiation conversion efficiency. The measurements carried out on the crop biomass including the crowns and the fraction of tap roots in the first 10 centimeters show that at least $80 \%$ of the consequences of water deficits consist of a modification of the dry matter partition between above and underground parts of the plants. In case of early water deficits, the restriction of the leaf area expansion results in a decrease of the total intercepted radiation, which appears to be the main cause of production deficits over the five studied regrowths.

water use - irrigation - leaf aera index - yield - forage - plant biomass

\section{Introduction}

Une des variables à l'origine des plus fortes variations de production de la luzerne durant la saison estivale, en climat tempéré, est le déficit hydrique (Robelin, 1969). La luzerne, malgré une exploration du sol en général profonde, peut voir sa croissance réduite en condition de déficit d'alimentation hydrique (Christian, 1977). Un déficit hydrique est susceptible d'affecter l'ensemble des fonctions physiologiques : photosynthèse, nutrition minérale, transports d'assimilats, morphogenèse (Bradford et Hsiao, 1982). Ces fonctions ne sont pas toutes également sensibles à la sécheresse (Merrien et al., 1981), de sorte que la vitesse de croissance de la plante peut être limitée davantage par l'une ou l'autre selon les conditions liées notamment à l'intensité de la sécheresse et à la position de celle-ci dans le cycle de croissance.

Une façon de prévoir l'effet de la sécheresse sur la production de matière sèche récoltable consiste à utiliser la corrélation entre la consommation hydrique et la production, ces 2 variables étant rapportées à leurs optimums respectifs. Cela a été développé par Robelin (1969) sous forme de la relation :

(MS en sec) / (MS potentielle) $=a \times E T R / E T M+b$

où MS est la matière sèche récoltée, ETR/ETM est le rapport entre les évapo-transpirations réelles et maximales et $a$ et $b$, des coefficients d'ajustements variant selon les espèces.

Cependant, la consommation en eau dépend de l'indice foliaire, qui lui-même dépend de la croissance. Ce type de modèle ne permet pas de 
déterminer l'origine du déficit de production, qui provient soit d'un défaut d'interception au rayonnement solaire, soit d'une répartition différente des assimilats entre parties aériennes et souterraines ou bien encure d'une réduction de la photosynthèse nette.

Gosse et al. (1984) ont proposé un modèle de production potentielle de luzerne, basé sur la relation entre la production de biomasse aérienne et la somme de rayonnement visible intercepté par le couvert végétal au cours de la repousse. $\mathrm{Ce}$ modèle permet de distinguer les phénomènes relevant de la morphogenèse et qui déterminent l'efficience d'interception (dynanisme d'expansion de la surface de feuilles) des phénomènes trophiques, qui se traduisent par l'efficience de conversion de l'énergie interceptée. II se présente sous la forme suivante :

$M S A=\varepsilon_{B} \times \varepsilon_{p} \times \Sigma\left[R g(j) \times 0,5 \times\left(1-e^{-0.88 \times I F(j)}\right)\right]$

où MSA est la matière sèche aérienne récoltée, $\varepsilon_{B}$ est l'efficience de conversion du rayonnement intercepté, $\varepsilon_{p}$ est le taux de répartition de la matière sèche entre parties aériennes et parties souterraines, $R g(j)$ est le rayonnement global du jour $j, \operatorname{IF}(j)$ est l'indice foliaire de la culture au jour $j$.

Analyser séparément les 2 composantes $\varepsilon_{B}$ et $\varepsilon_{p}$ nécessite donc de prendre en compte les variations de la biomasse souterraine.

L'objectif du présent article est d'illustrer une utilisation possible d'un tel modèle à partir de données agronomiques classiques, en vue d'analyser les effets de la sécheresse sur la production estivale de luzerne.

\section{Matériel et Méthodes}

\section{Conduite de la culture}

La culture (variété Europe) a été implantée en mai 1981 sur le site expérimental de Lusignan (Vienne). Le sol est un sol brun lessivé sur limon, superposé à une argile rouge à pisolithes. II est limoneux sur les 50 premiers centimètres et devient argilo-limoneux à partir de cette profondeur, avec des concrétions ferro-manganiques et des taches d'oxydo-réduction du fer (Chesseron, 1986). Le dispositif consistait en 4 blocs aléatoires complets comprenant 4 parcelles de $50 \mathrm{~m}^{2}$. Deux parcelles étaient irriguées et 2 autres conduites en sec. L'une des 2 parcelles a été utilisée en 1982 et l'autre en 1983. Les différentes opérations culturales conduites sur l'essai sont présentées sur le Tableau I.

Aucune irrigation n'a été réalisée en 1981. L'irrigation était réalisée par des asperseurs (de $90 \mathrm{~cm}$ de hauteur), distants les uns des autres d'un rayon de 5 $\mathrm{m}$. La dose appliquée à chaque fois a été de $40 \mathrm{~mm}$ au maximum. Les coupes, irrigations et divers travaux ont été réalisés selon le Tableau 1.

\section{Mesures météorologiques}

Les relevés météorologiques comportaient les mesures journalières des températures minima et maxima, des précipitations et du rayonnement global incident (pile de Kipp).

\section{Bilan hydrique simplifié}

Une mesure hebdomadaire d'humidimétrie neutronique (sonde Solo 20 ) était réalisée sur 8 profils de $1,80 \mathrm{~m}$, soit 4 profils par traitement. La procédure de mesure est décrite dans Lemaire et Roberge (1980).

\section{Mesures sur les végétaux}

Les courbes de croissance ont été réalisées à partir de prélèvements hebdomadaires de la matière sèche aérienne (dans la suite, MSA) sur chacune des répétitions ( $2 \times 4 \mathrm{~m}$ de ligne par traitement).

L'analyse morphologique du peuplement était réalisée au même moment sur un prélèvement plus limité : sur un mètre linéaire sur chaque traitement, toutes les plantes présentes étaient récoltées. Cet échantillon était séparé en 4 composantes : les feuilles $(F)$, les tiges $(T)$, les souches $(S)$, c'est-à-dire la partie aérienne non récoltée (de 0 à $6 \mathrm{~cm}$ ), et les pivots dans l'horizon 0-10 cm (R).

Les rapports entre les masses de feuilles et de tiges d'une part, masse des parties aériennes sur matière sèche totale de l'échantillon d'autre part permettaient de calculer la biomasse de chaque compartiment. Pour les feuilles:

$$
\text { MSfeuilles }=[F /(F+T)] \times \text { MSA }
$$

Dans la suite, la biomasse "totale" incluant souches et pivots sur $0-10 \mathrm{~cm}$ sera notée MSR :

$$
M S R=[(R+S+T+F) /(F+T)] \times M S A
$$

L'étude des variations de la biomasse des pivots avait pour but de mettre en évidence les éventuels transferts de réserves entre parties aériennes et parties souterraines. D'après des résultats de VarletGrancher (non publiés) et Durand (1987), l'essentiel des variations de biomasse souterraine ont lieu dans les 10 premiers centimètres, où se situent 65 et $70 \%$ de la biomasse comprise dans les 50 et 30 premiers centimètres respectivement. La limitation de l'extraction aux 10 premiers centimètres est due à la difficulté du prélèvement des racines dans les horizons plus profonds sur les parcelles en sec. II ne s'agit donc que d'une estimation par défaut de ces variations de biomasse, qui n'aura de signification qu'en valeur relative entre les traitements sec et irrigué.

\section{Résultats}

Le rapport (en pourcentage) entre les consommations d'eau en sec et en irrigué est représenté en fonction du temps pour les 2 années (Figures 1 et 2). Il permet une première quantification de l'effet de la sécheresse. Sur les 5 cycles, le rapport a varié entre 40 et $100 \%$ selon la période.

Sur les mêmes figures, les courbes de croissance de la MSA (en g.m-2) sont également indiquées. On constate des variations de production des traitements non irrigués allant de 14 à $90 \%$ 
Tableau I. Opérations culturales réalisées sur l'essai.

\begin{tabular}{|c|c|c|c|c|}
\hline Date & Semis, coupe & Fertilisation & Désherbage & Irrigation \\
\hline \multicolumn{5}{|l|}{1980} \\
\hline$\uparrow 4,11$ & & $80 \mathrm{U}, \mathrm{P} 205$ & & \\
\hline 22,12 & & $120 \mathrm{U}, \mathrm{K} 20$ & & \\
\hline \multicolumn{5}{|l|}{1981} \\
\hline 16,4 & Semis & & & \\
\hline 24,7 & Coupe & & & \\
\hline 11,9 & Coupe & & & \\
\hline 4,11 & Coupe & & & \\
\hline 19,11 & & & Kerb Ultra & \\
\hline 25,11 & & 80 U, P 205 & & \\
\hline 27,11 & & $120 \mathrm{U}, \mathrm{K} 20$ & & \\
\hline \multicolumn{5}{|l|}{1982} \\
\hline 19,5 & Coupe & & & \\
\hline 28,5 & & & & $40 \mathrm{~mm}$ \\
\hline 1,6 & & & & $27 \mathrm{~mm}$ \\
\hline 24,6 & Coupe & & & \\
\hline 26,6 & & & & $15 \mathrm{~mm}$ \\
\hline 30,6 & & & & $50 \mathrm{~mm}$ \\
\hline 12,7 & & & & $50 \mathrm{~mm}$ \\
\hline 22,7 & & & & $22 \mathrm{~mm}$ \\
\hline 29,7 & Coupe & & & \\
\hline 30,7 & & & & $10 \mathrm{~mm}$ \\
\hline 2,8 & & & & $05 \mathrm{~mm}$ \\
\hline 24,8 & & & & $50 \mathrm{~mm}$ \\
\hline 9,9 & Coupe & & & \\
\hline 16,9 & & & & $25 \mathrm{~mm}$ \\
\hline 28,10 & Coupe & & & \\
\hline \multicolumn{5}{|l|}{1983} \\
\hline 18,1 & & $120 \mathrm{U}, \mathrm{K} 20,90 \mathrm{U}, \mathrm{K} 20$ & & \\
\hline 25,1 & & Legurame + Kerb Ultra & & \\
\hline 27,5 & Coupe & & & \\
\hline 30,5 & Coupe & & & \\
\hline 21,6 & & & & $30 \mathrm{~mm}$ \\
\hline 22,6 & & & & $20 \mathrm{~mm}$ \\
\hline 6,7 & Coupe & & & \\
\hline 7,7 & & & & $50 \mathrm{~mm}$ \\
\hline 20,7 & & & & $30 \mathrm{~mm}$ \\
\hline 4,8 & & & & $25 \mathrm{~mm}$ \\
\hline 17,8 & Coupe & & & \\
\hline 5,10 & Coupe & & & \\
\hline
\end{tabular}

du traitement irrigué. Les périodes de faible croissance des parcelles en sec correspondent globalement aux périodes où la consommation en eau est restreinte.

En 1982 (Fig. 1), la première repousse a été affectée à partir du milieu du cycle (8 juin). Pour la deuxième repousse, la sécheresse s'est située dès le début de la repousse et, en moyenne, la consommation d'eau de la culture non irriguée est restée un peu inférieure à $50 \%$ de la consommation du témoin irrigué sur ce cycle. Pour la troisième repousse, une période de pluie totali- sant $60 \mathrm{~mm}$ a commencé juste après la deuxième coupe, et les croissances en sec et en irrigué ont été identiques durant les deux tiers de la repousse. A partir du 21 août, la consommation d'eau s'est restreinte à $45 \%$ de celle des parcelles irriguées et la croissance des parties aériennes en sec s'est annulée.

En 1983 (Fig. 2), l'irrigation du 30 mai en première repousse n'a pas créé de différences. Le rapport entre les consommations en eau est resté constamment proche de $100 \%$ et la vitesse de croissance est restée la même sur tout le 


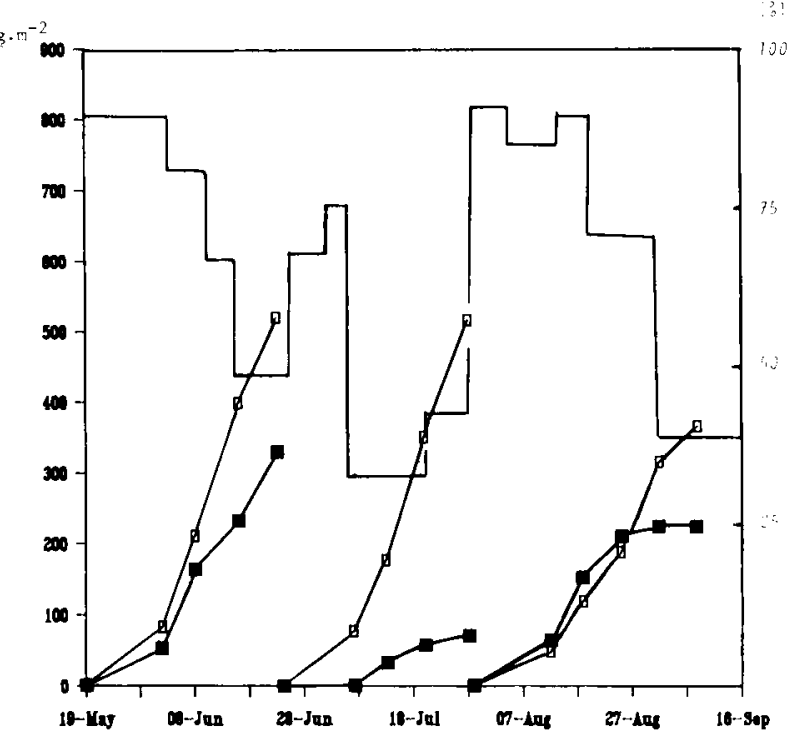

Fig. 1. Courbe de croissance de la matière sèche aérienne

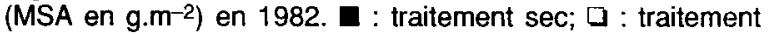
irrigué. Evolution du rapport entre les consommations en sec et en irrigué $\left(\mathrm{CH}_{\text {sed }} / \mathrm{CH}_{\text {irrigué }}\right)$ en pourcentage.

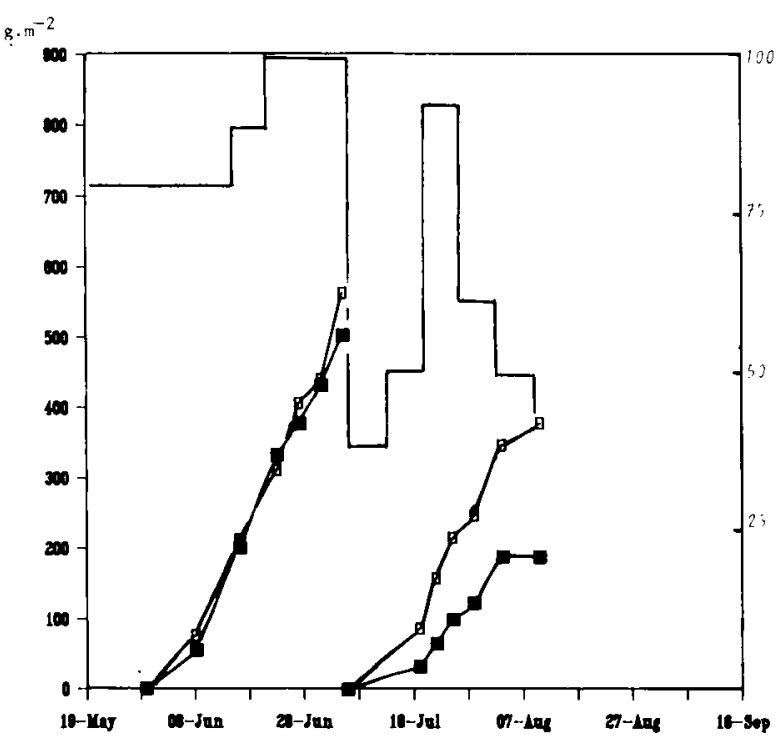

Fig. 2. Courbe de croissance de la matière sèche aérienne

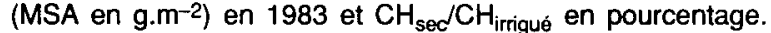
Q : traitement sec; $\square$ : traitement irrigué.

cycle. Dès le début de la deuxième repousse, la consommation en sec n'atteignait que $40 \%$ de la consommation en irrigué et la croissance était nettement plus faible sur les parcelles sèches.

Les Figures 3 et 4 indiquent les courbes d'évolution de la MSR pour les 5 cycles. Pour les repousses 2 et 3 en 1982 et pour la repousse 2 en 1983 et dans les 2 situations d'alimentation hydrique, la vitesse de croissance de la MSR durant les 10 premiers jours apparaît nulle ou très faible ou bien même négative. Cela alors que la croissance en matière sèche aérienne (MSA) est positive.

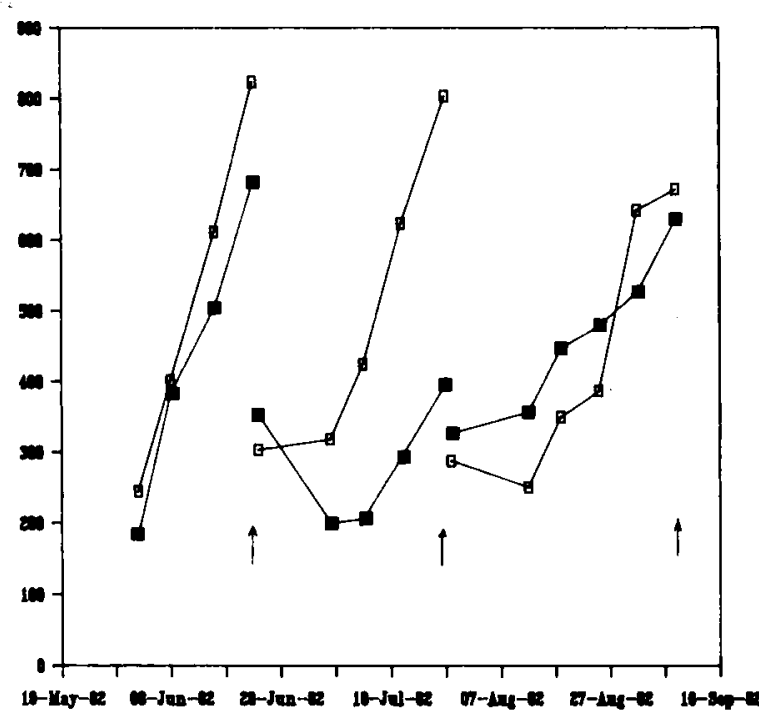

Fig. 3. Courbes de croissance de la matière sèche, y compris les souches et les pivots dans l'horizon 0-10 cm (MSR en g. $\mathrm{m}^{-2}$ ) en 1982. $\square$ : traitement sec; $\square$ : traitement irrigué.

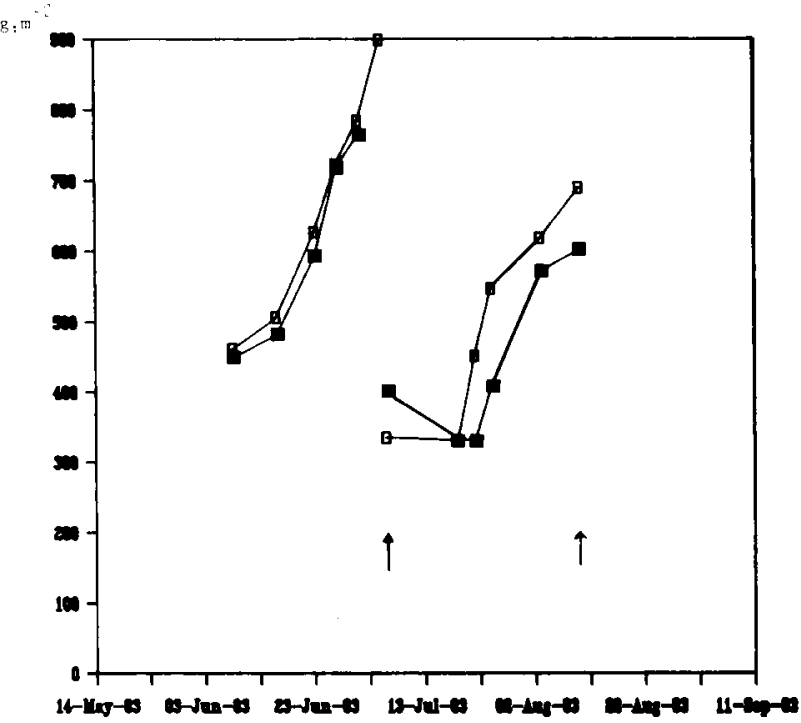

Fig. 4. Courbes de croissance de la matière sèche, y compris

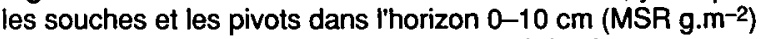
en 1983. I : traitement sec; $\square$ : traitement irrigué.

Le Tableau II présente les rapports entre les vitesses de croissance moyenne des 2 traitements pour chaque cycle pour MSA et MSR : [(dMS/dt)sec] / [(dMS/dt)irrigué], ainsi que les rapports entre les consommations hydriques (CH) en sec et en irrigué.

Ce tableau montre que, pour un rapport de consommation hydrique donné, la réduction de la production est toujours plus sensible pour la MSA que pour la MSR. Pour la seconde repousse de 1982 par exemple, la sécheresse a réduit la vitesse de croissance des parties aériennes de $85 \%$ et seulement de $59 \%$ en considérant la MSR. 
Tableau II. Rapport entre les vitesses de croissance moyenne en sec et en irrigué pour la MSA et la MSR, et rapport entre les consommations hydriques $\left(\mathrm{CH}_{\text {sed }} / \mathrm{CH}_{\text {irrigue }}\right)$, par repousse.

\begin{tabular}{llll}
\hline & MSA & MSR & $\mathrm{CH}_{\text {sed }} / \mathrm{CH}_{\text {irnigue }}$ \\
\hline 82.1 & 0,64 & 0,86 & 0,72 \\
82.2 & 0,15 & 0,41 & 0,39 \\
82.3 & 0,62 & 0,65 & 0,60 \\
83.1 & 0,90 & 1,12 & 0,95 \\
83.2 & 0,47 & 0,55 & 0,54 \\
\hline
\end{tabular}

\section{Discussion}

Vitesses de croissance de la biomasse incluant les pivots et les souches (MSR) comparée à la biomasse aérienne seule (MSA)

La quantité relative de matière sèche allouée aux parties non récoltées a eu tendance à augmenter dans le traitement sec. II s'agit du résultat d'une moindre sensibilité de la croissance racinaire à la sécheresse, comparée à la croissance des parties aériennes. Un tel processus a été décrit par Abdul-Jabbar et al. (1982) sur les racines fines (les pivots n'avaient pas été pris en compte dans leur analyse). De même, Cohen et al. (1972) et Durand (1987) ont montré que la sécheresse induisait une augmentation du taux de sucres solubles dans les pivots. On confirme ici que les quantités ainsi mises en jeu peuvent être assez importantes par rapport aux différences de niveau de production des parties aériennes.

La phase de croissance précoce nulle ou négative observée sur les Figures 3 et 4 coïncide avec la remobilisation des réserves contenues dans les souches et les pivots (Pearce et al., 1969; Ueno et Smith, 1970). La source de carbone pour la croissance aérienne est alors double (Demarly, 1957; Gosse et al., 1982). II s'agit, d'une part, de la photosynthèse des feuilles se mettant en place progressivement, et des sucres de réserves des pivots et souches d'autre part. Tout se passe comme si, sur chaque repousse, le point de matière sèche totale minimum se situait à la date où la satisfaction de la demande en carbone des parties aériennes (respiration comprise) devenait inférieure à l'offre de la photosynthèse du couvert. Le fait que la MSR chute entre la coupe et le premier prélèvement indique une perte nette de carbone. Soit une part importante de la biomasse est allouée aux parties du système racinaire non prises en compte dans l'estimation de la biomasse totale, soit cette perte est entièrement expliquée par la respiration. Un résultat semblable avait été signalé par Ueno et
Smith (1970), mais sur des plantes isolées, en pot et seulement pour les individus de grande taille. Il est intéressant de constater à l'échelle de la parcelle également une phase de reprise de végétation correspondant à un bilan de biomasse négatif.

L'idéal aurait été de mesurer effectivement les variations de la biomasse totale présente sur la parcelle, c'est-à-dire de mesurer la totalité de la biomasse racinaire. Khaity (1985) a montré que ces variations liées à la coupe n'étaient perceptibles que sur les 25 premiers centimètres. Enfin, la bibliographie indique que la partie inférieure du pivot suit une cinétique semblable à celle de la partie supérieure, que nous prenons seule en compte (Escalada et Smith, 1972; Rappoport et Travis, 1984).

Analyse de l'efficience de conversion du rayonnement par un couvert de luzerne subissant une sécheresse

La sécheresse peut avoir diminué la vitesse de croissance de la MSA soit en réduisant la quantité d'énergie interceptée (lorsque l'indice foliaire est limitant), soit en réduisant la part allouée aux parties aériennes $\left(\varepsilon_{p}\right)$, soit en affectant la conversion de l'énergie interceptée en biomasse totale $\left(\varepsilon_{\mathrm{B}}\right)$.

\section{Estimation de la quantité d'énergie interceptée au cours d'un cycle}

La relation (2) fournit un moyen d'estimer la quantité d'énergie interceptée pour chaque jour. Le rayonnement global a été relevé quotidiennement. La mesure de l'indice foliaire n'ayant pas été faite directement, son estimation a été réalisée à partir de la biomasse de feuilles, en lui affectant une masse surfacique moyenne. Nous avons supposé qu'en irrigué la masse surfacique des feuilles était en moyenne de $35 \mathrm{~g} \cdot \mathrm{m}^{-2}$ au long d'un cycle. Cette valeur correspond aux nombreuses mesures effectuées par ailleurs sur le même site et à la même saison (Cruz et Lemaire, 1986; Khaity, 1985). Pour les plantes non irriguées et afin de prendre en compte d'éventuelles variations induites par la sécheresse, nous avons estimé pour chaque date 2 indices foliaires correspondant à 2 valeurs extrêmes de masse surfacique : 30 et $45 \mathrm{~g} \cdot \mathrm{m}^{-2}$ (Pearce et al., 1969; Smith et Struckmeyer, 1974; Hart et al., 1978; Durand, 1987). Pour maximiser l'écart, ce calcul a été réalisé sur l'ensemble du cycle, quel qu'ait été le ratio entre les consommations hydriques. L'écart maximum correspondant à ces valeurs extrêmes n'a été ainsi que de $12 \%$.

L'estimation journalière de l'indice foliaire entre 2 points de mesures a été réalisée en utili- 
sant une relation linéaire entre indice foliaire et somme de température (Gosse et al., 1984).

Relation entre matière sèche et somme de rayonnement pour les témoins irrigués

La Figure 5 représente les relations entre MSA et MSR d'une part et la somme de rayonnement intercepté d'autre part, pour l'ensemble des repousses en conditions hydriques non limitantes. La pente est de 1,72 g.MJ-1 pour la matière sèche aérienne, ce qui est très proche de 1,76 g.MJ-1, valeur donnée par Gosse et al. (1984). Il apparaît par ailleurs que les différences de production entre coupes ne sont attribuables qu'à une différence de quantité de rayonnement intercepté, sauf pour la fin de la deuxième repousse de 1983, dont la productivité apparaît nettement plus faible que celle prévue par le modèle.

Pour MSR, la pente moyenne est de 2,3 g.MJ-1. Cette valeur est du même ordre de grandeur que celle donnée par Gosse et al. (1986) et Charles-Edwards et al. (1986) pour des espèces très variées. Alcock et Al-Juboury (1981) donnent une valeur de 2,4 g.MJ-1 pour la biomasse totale d'une fétuque élevée.

La pente de 1,76 g.MJ-1 représente l'efficience d'accumulation de la matière sèche dans les parties aériennes, en saison estivale, au cours d'une repousse avec irrigation. La pente de 2,3 g.MJ-1 représente réellement l'efficience de conversion de l'énergie en biomasse $\left(\varepsilon_{B}\right)$. Le rapport entre ces 2 pentes représente donc le coefficient de répartition entre les parties récoltées et non récoltées, soit 0,76 (relation (2)). Autrement

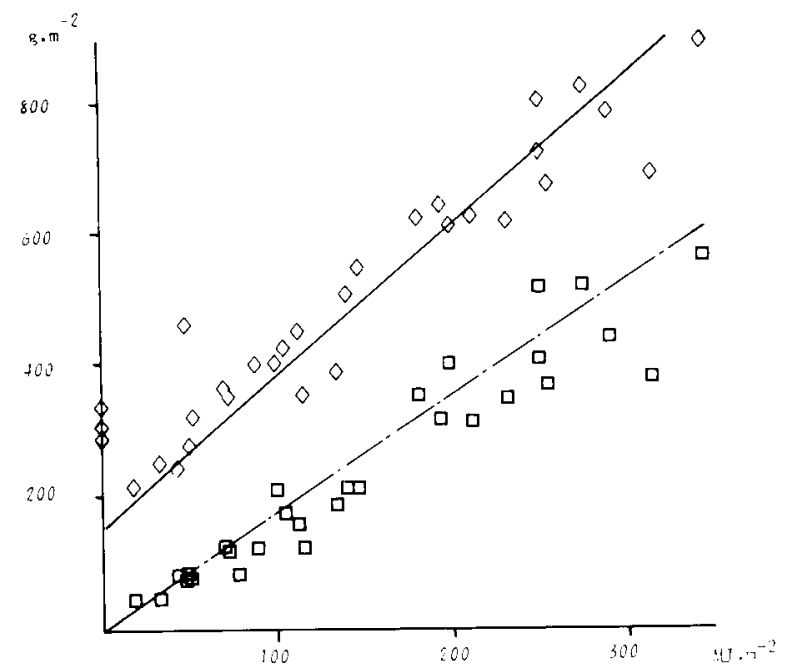

Fig. 5. Matière sèche en g.m $\mathrm{m}^{-2}$ produite dans les cycles irrigués à Lusignan (1982 et 1983) en fonction de la somme de rayonnement visible intercepté en $\mathrm{MJ} . \mathrm{m}^{-2}$. $\square$ : matière sèche aérienne; : matière sèche y compris les souches et les pivots de l'horizon 0-10 $\mathrm{cm}$ (MSR); - régression $\alpha$, pour la matière sèche $y$ compris les pivots et les souches : MSR $=2,3 \times \Sigma R i+150 ; R^{2}=0,894$ - - - - : régression $\beta$, pour la matière sèche aérienne : $M S A=1,76 \times \Sigma R i ; R^{2}=0,960$ dit, les trois quarts de la matière sèche résultant de la conversion de l'énergie solaire visible interceptée sont consacrés à l'élaboration de tiges et de feuilles.

L'efficience de 2,3 g.MJ-1 ne s'applique qu'à la biomasse nouvellement élaborée après la coupe et prend en compte les pertes respiratoires de croissance et d'entretien qui lui sont liées. En revanche, les pertes respiratoires de la biomasse résiduelle après la coupe ne sont pas intégrées dans cette relation. Après la coupe, il y a donc 2 cinétiques différentes à considérer :

- la cinétique d'accumulation de nouveaux matériaux qui s'effectue en fonction du rayonnement intercepté, avec une efficience de conversion constante de 2,3 g.MJ'1;

- la cinétique des pertes respiratoires liées au matériel végétal restant après la coupe et qui, chaque jour, sont proportionnelles à la partie restante de cette biomasse.

Le schéma d'interprétation de la Figure 6 montre les 2 cinétiques et leur résultante, qui correspond à l'évolution de la biomasse totale.

La différence entre la biomasse restante après la coupe (point $A$ ) et l'ordonnée à l'origine de la relation $M S R=f($ Rint) (point $B$ ) représente donc la part de la biomasse résiduelle qui a été perdue lors de la repousse.

En prenant la valeur de 300 g.m-2 comme valeur moyenne de $A$ et en supposant un coefficient de respiration d'entretien de cette matière sèche situé entre 1 et $3 \%$ (Ruget, 1981), on peut estimer que la quantité de carbone utilisée pour l'entretien durant une période moyenne de 15 jours correspond à une quantité de matière sèche comprise entre 40 et $110 \mathrm{~g} \cdot \mathrm{m}^{-2}$, ce qui est

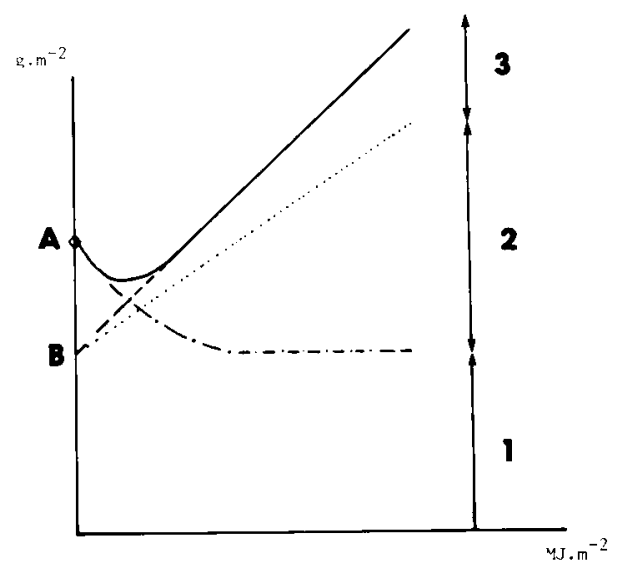

Fig. 6. Schéma représentant les relations théoriques entre la MSR ( $\longrightarrow$, le matériel vivant provenant de la coupe précédente et non récolté (- - _- - ), les régressions $\alpha(--$ $-\rightarrow$ et $\beta(. . .$.$) en fonction de la somme de rayonnement$ intercepté. 1 correspond à la part de la MSR reportée de la coupe précédente, 2 représente la croissance aérienne, et 3 la part de la matière sèche nouvellement affectée aux parties non récoltées. La somme $1+2+3$ est égale à la MSR. 
du même ordre de grandeur que les valeurs effectivement observées (A-B).

\section{Analyse des effets de la sécheresse}

La comparaison systématique aux témoins irrigués des croissances en conditions sèches est présentée Figure 7. Sur chaque graphe, les 2 droites de référence correspondant à une alimentation hydrique non limitante ont été tracées. Pour la relation entre la MSR et la somme de rayonnement intercepté, l'ordonnée à l'origine $(A)$ peut être différente d'une repousse à l'autre du fait des processus décrits précédemment. Les comparaisons avec les traitements irrigués ont été faites en utilisant une droite de pente 2,3 g.MJ-1 et passant par le point moyen.

La réduction de la quantité d'énergie interceptée a eu un effet réellement mesurable (étant donné l'erreur d'estimation de l'indice foliaire en sec) sur la deuxième repousse de 1982 et la deuxième repousse de 1983 (Fig. 7b et d). Dans ces 2 cas, l'intervention précoce de la sécheres-

a

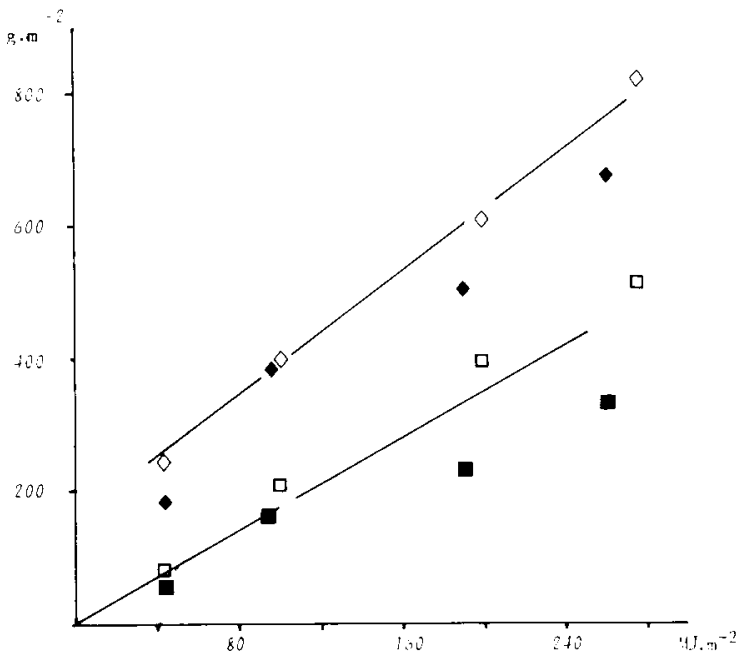

c

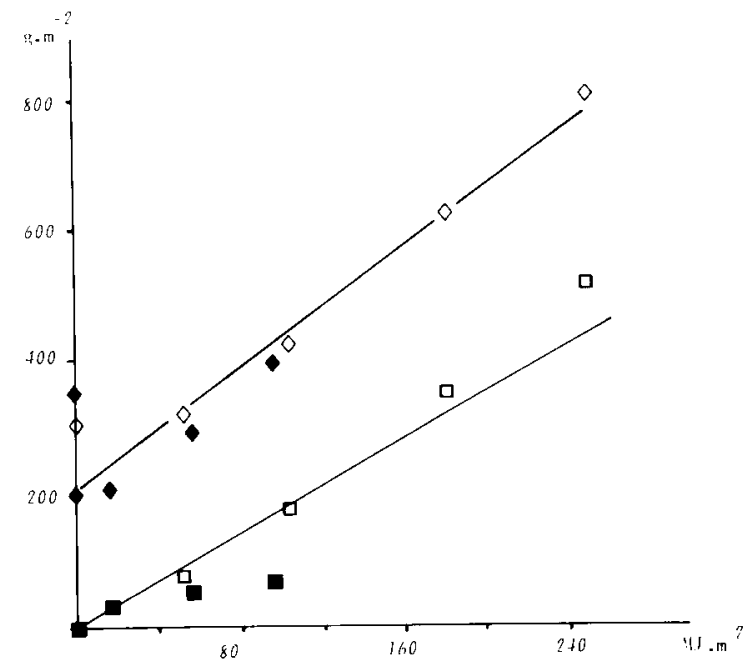

se a limité immédiatement la vitesse de croissance des feuilles. Les conséquences de cette limitation de la quantité totale d'énergie interceptée expliquent respectivement de l'ordre de 60 et $20 \%$ du déficit de production du traitement sec par rapport au témoin irrigué.

Pour les 4 repousses où la sécheresse s'est manifestée, à même quantité d'énergie interceptée, la MSA a été davantage affectée que la MSR. Cela signifie que le coefficient de répartition des assimilats entre les parties aériennes et les parties souterraines $\left(\varepsilon_{p}\right)$ est plus sensible à la sécheresse que l'efficience de conversion de l'énergie interceptée en matière sèche $\left(\varepsilon_{\mathrm{B}}\right)$.

Pour l'ensemble de la deuxième repousse de 1982 (Fig. 7b), le couvert non irrigué a converti l'énergie interceptée en MSA avec une efficience moyenne atteignant seulement $20 \%$ de celle du couvert irrigué, alors que les $\varepsilon_{B}$ (concernant la MSR) ont été identiques et égaux à l'efficience potentielle de 2,3 g.MJ-1. Pour la deuxième repousse de 1983 (Fig. 7d), il n'est pas possible
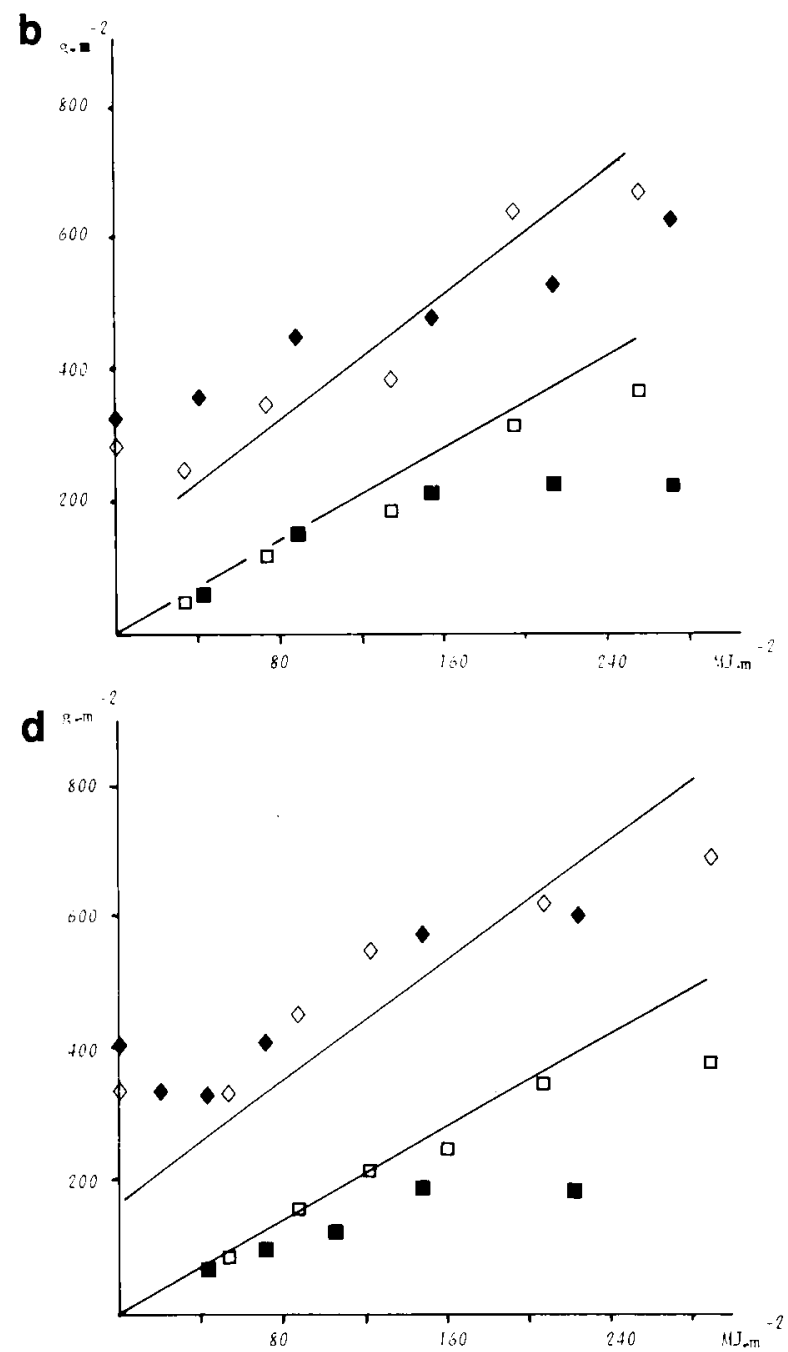

Fig. 7. Pour chaque cycle, matière sèche en $\mathrm{g} . \mathrm{m}^{-2}$ en fonction de la somme de rayonnement intercepté en $\mathrm{MJ} \cdot \mathrm{m}^{-2}$, et régressions A et B. $\square$ : matière sèche aérienne, irrigué; $\diamond:$ matière sèche, y compris souches et pivots, irrigué; $\square$ : matière sèche aérienne, sec; $\checkmark$ : matière sèche, y compris souches et pivots, sec; $\_$: régressions $\alpha$ et $\beta$; $a: 1982,1$ re repousse, b : 1982, $2^{\circ}$ repousse, c : $1982,3^{\theta}$ repousse, $d: 1983,2^{\theta}$ repousse. 
de mettre en évidence une différence de $\varepsilon_{\mathrm{B}}$ entre les traitements, mais l'un et l'autre ont présenté une efficience inférieure à l'efficience potentielle. La cause de cette faible valeur n'a pu être établie avec certitude.

En 1982, au cours de la troisième repousse (Fig. 1), la sécheresse a conduit à une réduction de la consommation en eau du même ordre de grandeur qu'au cours de la deuxième. En revanche, les effets sur la production ont été très différents : en deuxième repousse, la sécheresse a été précoce et a conduit à une réduction plus importante de la production, tandis qu'en troisième repousse la sécheresse a été tardive et l'influence sur la croissance plus réduite. Comme le montre la Figure $7 b$, la sécheresse précoce sur la deuxième repousse a eu essentiellement comme conséquence une réduction de la quantité de rayonnement intercepté, mais sans aucune diminution de $\varepsilon_{\mathrm{B}}$. En revanche, la sécheresse tardive de la troisième repousse intervenant sur un couvert déjà bien développé n'a pas diminué la quantité de rayonnement intercepté et a réduit $\varepsilon_{B}$ (Fig. 7c).

La comparaison de ces 2 exemples montre que la détermination de l'intensité de la sécheresse par les rapports de consommation en eau en sec et en irrigué n'est pas pertinente du point de vue du fonctionnement du peuplement végétal. II aurait fallu disposer de mesures de l'état hydrique du couvert végétal (potentiel hydrique foliaire, par exemple). En l'absence de cette information, on peut proposer 2 hypothèses explicatives.

Lors de la sécheresse précoce (deuxième repousse de 1982), seule une proportion très faible de plantes a émis des tiges et feuilles vertes. On peut alors supposer que ces plantes, grâce à un système racinaire plus profond et plus développé que la moyenne de la population, se trouvaient dans un état hydrique peu limitant pour leur morphogenèse. Cela pourrait expliquer l'absence d'effet sur $\varepsilon_{B}$, la photosynthèse des feuilles n'ayant pas été affectée à cet état hydrique. Cette hypothèse est corroborée par l'observation qui a été faite d'une très grande hétérogénéité microspatiale. En revanche, la sécheresse tardive de la troisième repousse intervenant soudainement sur un couvert bien développé a pu affecter l'état hydrique de la totalité du peuplement, ce qui s'est traduit par une diminution de $\varepsilon_{\mathrm{B}}$.

A cette première hypothèse, on peut ajouter l'éventualité d'une adaptation progressive des plantes. Une sécheresse rapide, telle que celle qui s'est établie au cours de la troisième repousse de 1982, a pu davantage affecter la capacité développées en conditions hydriques optimales (Matthews et Boyer, 1984). Au contraire, les feuilles de la seconde repousse de 1982 ont connu des conditions sèches au cours de leur développement et de ce fait ont pu s'y adapter, maintenant globalement la valeur $d^{\prime} \varepsilon_{B}$.

\section{Conclusion}

La sécheresse semble affecter principalement les processus qui déterminent la morphogenèse des parties aériennes. Une réduction de la croissance des feuilles en début de repousse, quand l'indice foliaire est inférieur à 3 , conduit à une diminution sensible de la quantité de rayonnement intercepté. Cela se traduit par une diminution de croissance, sans que les processus de conversion de l'énergie captée ni ceux de la répartition des assimilats soient nécessairement affectés. Une réduction de la croissance des tiges feuillées peut se traduire par une augmentation de la biomasse racinaire correspondant approximativement à la diminution observée des parties aériennes. Dans ce cas, les mécanismes liés à la conversion de l'énergie peuvent ne pas avoir été affectés.

Dans le cadre de ces essais, l'effet du déficit hydrique sur la morphogenèse des parties aériennes explique entre 80 et $100 \%$ des différences de production en biomasse aérienne, que ce soit à travers des différences de quantité d'énergie interceptée ou bien à travers des différences de répartition d'assimilats. Les processus concernant le bilan de carbone (photosynthèse nette, respiration) ne semblent avoir été affectés que lors d'une sécheresse importante et sur un couvert bien développé. En revanche, en début de repousse, la sécheresse limite essentiellement la croissance foliaire et donc l'interception de rayonnement. Ces actions différentes du déficit hydrique selon le stade de repousse de la luzerne montrent qu'une relation linéaire univoque entre les consommations d'eau relatives et les croissances relatives ne peut rendre compte de manière prédictible l'effet d'une sécheresse. Il s'agit d'une nouvelle illustration de la nécessité de mieux connaître l'état hydrique du végétal ainsi que la sensibilité des différents processus à la sécheresse pour expliquer les variations de production dans des conditions variées. Ce type d'approche plus analytique (Robelin, 1984) décrit l'effet d'un déficit hydrique à partir de son expression au niveau de la plante. L'évolution de chacun des processus essentiels y est décrite en fonction de l'évolution de l'état hydrique de la plante (mesuré par son potentiel hydrique ou sa teneur en eau relative, par exemple) à l'échelle de l'heure ou de la journée. 
Ce type de démarche devrait permettre de mieux comprendre les effets de sécheresses se situant dans différentes situations.

\section{Références}

Abdul-Jabbar A.S., Sammis T.W. \& Lugg D.G. (1982) Effect of moisture level on the root pattern of alfalfa. Irrig. Sci. 3, 197-207

Alcock M.B. \& Al-Juboury H.H. (1981) The effect of solar radiation and water stress on the growth of grass. In: Plant Physiology and Herbage Production (Wright C.E., ed.). British Grassland Society, occasional symposium 13, pp. 225-230

Bradford K.J. \& Hsiao T.C. (1982) Physiological responses to moderate water stress. In: Encyclopedia of Plant Physiology. Physiological Plant Ecology II. Water Relations and Carbon Assimilation. Springer Verlag

Charles-Edwards D.A., Doley D. \& Rimmington G.M. (1986) Modelling Crop Growth and Development. Academic Press

Chesseron C. (1986) Contribution à l'étude des relations sol-plante. Lupin et luzerne dans les terres rouges à châtaigners du Poitou-Charentes. Thèse Sci., Poitiers

Christian K.R. (1977) Effects of environment on the growth of alfalfa. Adv. Agron. 29, 183-227

Cohen Y., Bielorai H. \& Dovrat A. (1972) Effect of timing of irrigation on total nonstructural carbohydrates level in roots and on seed yield of alfalfa (Medicago sativa L.). Crop. Sci. 12, 634-636

Cruz P. \& Lemaire G. (1986) Analyse des relations de compétition dans une association de luzerne (Medicago sativa L.) et de dactyle (Dactylis glomerata L.). 1. Effet sur les dynamiques de croissance en matière sèche. Agronomie 6, 727-734

Demarly Y. (1957) Biologie et exploitation de la luzerne. Ann. Amelior. Plant. (Paris) 3, 247-272

Durand J.L. (1987) Modélisation de la production de luzerne et alimentation hydrique. Thèse de DocteurIngénieur

Escalada J.A. \& Smith D. (1972) Changes in nonstructural carbohydrate fractions at intervals down the tap root bark and wood of alfalfa (Medicago sativa L.) during regrowth. Crop. Sci. 12, 745-749

Gosse G., Chartier M., Lemaire G. \& Guy P. (1982) Facteurs climatiques et production chez la luzerne. Fourrages 90, 113-135
Gosse G., Chartier M. \& Lemaire G. (1984) Mise au point d'un modèle de prévision de production pour une culture de luzerne. C.R. Acad. Sci. Ser. III Sci. Vie 298, 541-544

Gosse G., Varlet-Grancher C., Bonhomme R., Chartier M., Allirand J.M. \& Lemaire G. (1986) Production maximale de matière sèche et rayonnement solaire intercepté par un couvert végétal. Agronomie 6, 47-56

Hart R.H., Pearce R.B., Chatterton N.J., Carlson G. E., Barnes D.K. \& Hanson C.H. (1978) Alfalfa yield, specific leaf weight, $\mathrm{CO}_{2}$ exchange rate and morphology. Crop Sci. 18, 649-653

Khaity M. (1985) Cinétique de croissance comparée des parties aériennes et souterraines de la luzerne (Medicago sativa L.). Effet d'une sélection sur la vigueur des pivots. Mémoire de DEA Sci., Poitiers

Lemaire G. \& Roberge C. (1980) Mise au point d'un dispositif expérimental permettant de suivre la consommation hydrique d'une prairie au cours de sa croissance. Ann. Agron. 31, 455-471

Matthews M.A. \& Boyer J.S. (1984) Acclimatation of photosynthesis to low leaf water potentials. Plant Physiol. 74, 161-166

Merrien A., Blancher R. \& Gelfi N. (1981) Relationships between water supply, leaf area development and survival and production in sunflower (Helianthus annuus L.). Agronomie 1, 917-922

Pearce R.B., Carlson G.E., Barnes D.K., Hart R.H. \& Hanson C.H. (1969) Specific leaf weight and photosynthesis in alfalfa. Crop. Sci. 9, 423-425

Rappoport H.F. \& Travis R.L. (1984) Alfalfa root growth, cambial activity and carbohydrate dynamics during the regrowth cycle. Crop. Sci. 24, 899-903

Robelin M. (1969) L'alimentation en eau des plantes fourragères. Fourrages $38,30-40$

Robelin M. (1984) Fonctionnement hydrique et adaptation à la sécheresse. In: Physiologie du Maïs. INRA, pp. 445-502

Ruget F. (1981) Respiration de croissance et respiration d'entretien : méthodes de mesures, comparaisons des résultats. Agronomie 1, 601-610

Smith D. \& Struckmeyer B.E. (1974) Gross morphology and starch accumulation in leaves of alfalfa plants grown at high and low temperatures. Crop Sci. 14, $433-436$

Ueno M. \& Smith D. (1970) Growth and carbohydrate changes in the root wood and bark of different sized alfalfa plants during regrowth after cutting. Crop Sci. 10, 396-399 